\title{
Bronchial Obstruction Caused by a Dilated Pulmonary Artery
}

\author{
Gurkirat Sandhu $^{1}$, Dikshya Sharma ${ }^{1}$, Kartikeya Rajdev ${ }^{2}$, Saad Habib ${ }^{1}$, Dany El-Sayegh ${ }^{3}$
}

1. Internal Medicine, Staten Island University Hospital, Northwell Health, Staten Island, USA 2. Pulmonary and Critical Care, University of Nebraska Medical Center, Omaha, USA 3. Pulmonary Critical Care, Staten Island University Hospital, Northwell Health, Staten Island, USA

Corresponding author: Gurkirat Sandhu, gurkiratss@hotmail.com

\begin{abstract}
Airway obstruction from an enlarged pulmonary artery (PA) is not a common occurrence. We present a rare case of respiratory failure secondary to right bronchus obstruction from a dilated right PA. A 54-year-old male with a known history of pulmonary hypertension $(\mathrm{PH})$ and obstructive sleep apnea (OSA) presented with worsening dyspnea. He was found to have collapse of his right middle and lower lobes. Intubation was required for respiratory failure. To our knowledge, this is the first case to be reported in the literature where PH caused PA dilatation to such a degree as to cause bronchial obstruction and subsequent lobar collapse.
\end{abstract}

Received 07/16/2019

Review began 08/02/2019 Review ended 08/06/2019 Published 08/09/2019

○) Copyright 2019

Sandhu et al. This is an open access article distributed under the terms of the Creative Commons Attribution License CC-BY 3.0., which permits unrestricted use, distribution, and reproduction in any medium, provided the original author and source are credited.
Categories: Internal Medicine, Pulmonology

Keywords: dilated pulmonary artery, pulmonary hypertension, bronchial obstruction, respiratory failure

\section{Introduction}

Pulmonary arteries (PA) can become dilated from pulmonary hypertension (PH). It has been well reported that this dilatation can cause compression of local structures such as the left main coronary artery or left recurrent laryngeal nerve [1-3]. Although less common, there are also reported cases of this dilatation resulting in airway compression. This is more often observed in pediatric populations because the bronchial tree of infants is underdeveloped and weak. There have been 10 cases reported that occurred in adults [4-9]. None of the previously reported cases presented with respiratory failure requiring mechanical ventilation.

We present a rare case, the first to our knowledge, of lobar collapse secondary to bronchial obstruction caused by PA dilatation from PH.

\section{Case Presentation}

A 54-year-old male presented with worsening dyspnea and bilateral lower extremity edema of a few days duration. His medical history was significant for PH, obstructive sleep apnea (OSA) on noninvasive ventilation (NIV), and obesity. Chest X-ray (CXR) in our emergency room was consistent with right middle and lower lobe collapse (Figure 1). Subsequent computed tomography (CT) of the chest revealed complete collapse of his right middle and lower lobes with suspicion of external compression (Figure 2). The right PA was noted to be markedly enlarged, twice the size of the aorta (Figure 3).

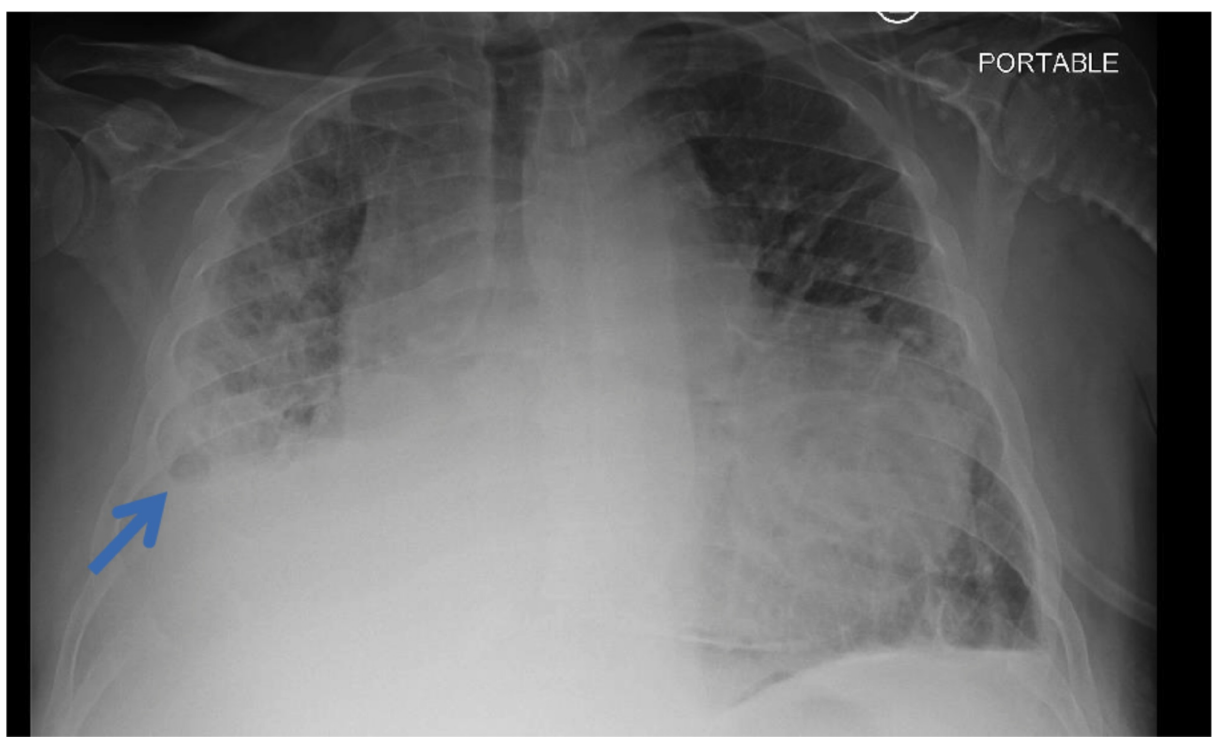




\section{Cureus}

FIGURE 1: Chest X-ray (CXR) showing collapse of right middle and lower lobes



FIGURE 2: Computed tomography (CT) scan of the chest (lung window) showing total occlusion of right middle and lower lobe bronchus

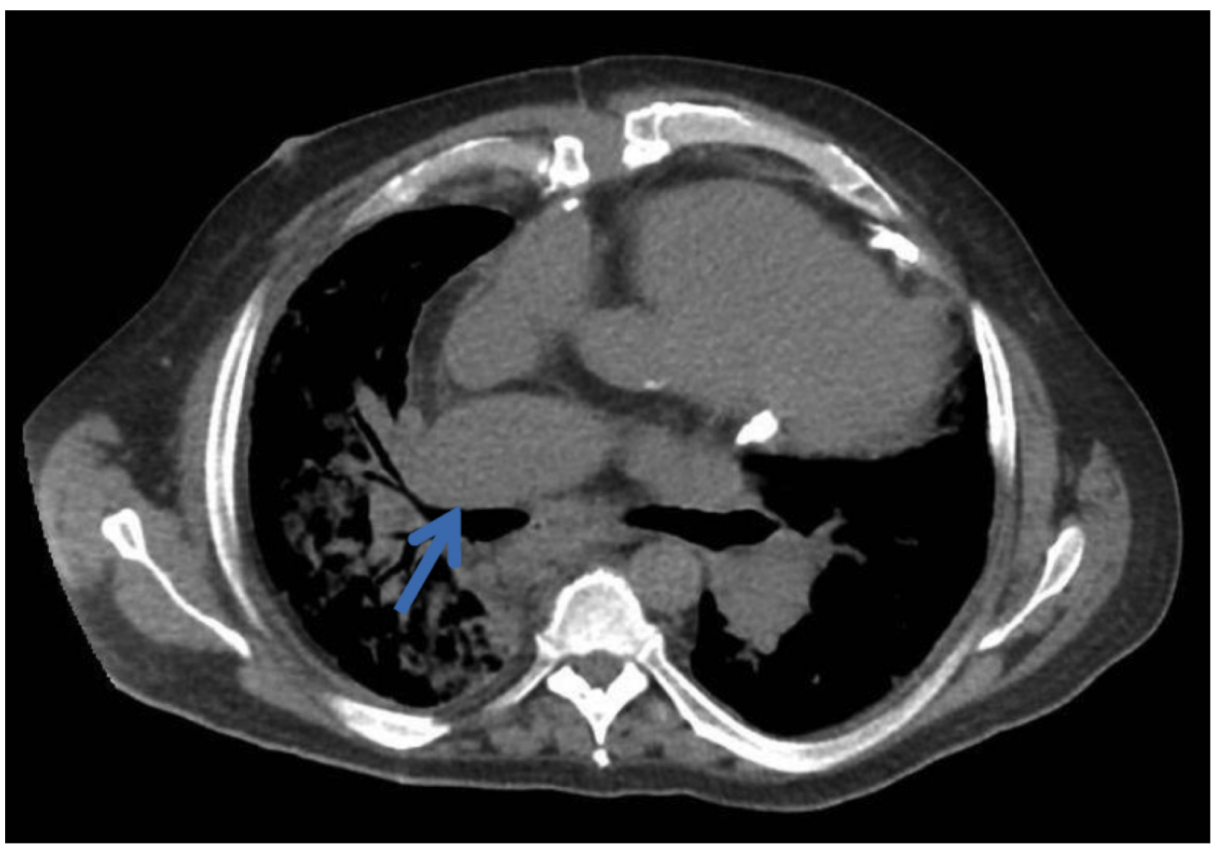

FIGURE 3: Computed tomography (CT) scan of the chest (abdomen window) showing enlarged right pulmonary artery (PA) causing external compression of right bronchus 
that were suctioned out. No endobronchial lesion was visualized. Extrinsic compression of the middle and lower bronchi was noted. Repeat CXR after bronchoscopy showed improvement of his right middle and lower lobe collapse. He was subsequently extubated the next day. Shortly after extubation, the patient again developed respiratory distress requiring continuous NIV support. Repeat CXR showed recurrent collapse of the right middle and lower lobes. A transthoracic echo was performed and showed a right ventricular systolic pressure of greater than $90 \mathrm{mmHg}$ with dilation of the right ventricle and moderate tricuspid regurgitation. His left sided ejection fraction was normal. The recurrent collapse of his right middle and lower lobes was attributed to the external compression of his bronchus from his enlarged right PA. He was subsequently transferred to a $\mathrm{PH}$ specialization center for further management.

\section{Discussion}

PA dilatation from PH is a well-documented phenomenon. When present, it has been shown to be an independent risk factor for death [10]. Bronchial obstruction caused by PA dilatation, however, is rare. Our literature review revealed 10 cases of airway obstruction from PA dilatation reported in adults [4-9]. Our case is unique amongst those previously reported in that the PA dilatation resulted in lobar collapse and subsequent respiratory failure. This was not described in any other case.

Airway obstruction from neighboring vascular structures is not commonly seen in adults. It is reported more frequently in pediatric populations. The tracheobronchial tree of infants is more susceptible to compression because the cartilaginous, muscular, and elastic support of airways is underdeveloped and weak. It has been reported in infants with tetralogy of fallot, absent pulmonary valve syndromes, atrial septal defects, and vascular rings such as double aortic arch, among others [11-13]. Acquired airway obstructions when present in adults usually arise from aortic aneurysms [11]. This is most often seen in the setting of atherosclerosis, although it can also be seen in connective tissue disorders such as Marfan's [14].

Patients with airway obstruction caused by PA dilatation most commonly present with dyspnea or wheezing. Sometimes, these patients can be misdiagnosed with late-onset asthma that is refractory to standard bronchodilator therapy [6].

In addition to airway obstruction, a dilated PA can also cause compression of other local structures. If the left main coronary artery is involved, it can cause myocardial ischemia that can mimic angina [3]. Alternatively, left recurrent laryngeal artery involvement can cause voice hoarseness [2].

CXR in patients with airway obstruction from PA dilatation can reveal a widened upper mediastinum. CT scan of the chest is the recommended diagnostic modality, preferably a CT angiogram with pulmonary embolus protocol study, which would reveal dilated pulmonary vasculature and compression of adjacent airways. A flexible bronchoscopy would reveal external compression of the bronchial tree. Magnetic resonance angiogram could also be used [15]. Spirometry may show flattening of the expiratory portion of the flow-volume curve [16].

Treatment of these patients can be challenging. PH specific treatment can reduce intravascular pressure but is unlikely to reduce vessel diameter. As such, airway obstruction would persist. Airway stenting, although possible, is not routinely employed. A high-tension stent would be required to keep airways open, creating concern for bronchial wall ischemia and erosion of the stent into surrounding structures [17-18]. Although there have been reported cases of successful stents deployed in infant populations [12], no such cases were found in adults. Surgical interventions are usually not considered in patients with severe PH as they are considered high operative risk [19]. Due to the limited treatment options, patients are typically referred to specialized centers.

\section{Conclusions}

We present a rare case, the first to our knowledge, of lobar collapse secondary to bronchial obstruction caused by PA dilatation from PH. The obstruction was seen on CT scan and confirmed with bronchoscopy. The patient required mechanical ventilation for respiratory failure with collapse of his right middle and lower lobes. He was transferred to a PH specialization center for further management. In patients with known PH and PA dilatation presenting with worsening dyspnea, airway obstruction should be considered as a possible differential diagnosis.

\section{Additional Information \\ Disclosures}

Human subjects: Consent was obtained by all participants in this study. Conflicts of interest: In compliance with the ICMJE uniform disclosure form, all authors declare the following: Payment/services info: All authors have declared that no financial support was received from any organization for the submitted work. Financial relationships: All authors have declared that they have no financial relationships at present or within the previous three years with any organizations that might have an interest in the submitted work. Other relationships: All authors have declared that there are no other 


\section{References}

1. Dakkak W, Tonelli AR: Compression of adjacent anatomical structures by pulmonary artery dilation . Postgrad Med. 2016, 128:451-459. 10.1080/00325481.2016.1157442

2. Heikkinen J, Milger K, Alejandre-Lafont E, et al.: Cardiovocal syndrome (Ortner's syndrome) associated with chronic thromboembolic pulmonary hypertension and giant pulmonary artery aneurysm: case report and review of the literature. Case Rep Med. 2012, 1-5. 10.1155/2012/230736

3. Lee J, Kwon HM, Hong BK, et al.: Total occlusion of left main coronary artery by dilated main pulmonary artery in a patient with severe pulmonary hypertension. Korean J Intern Med. 2001, 16:265-269. 10.3904/kjim.2001.16.4.265

4. Edwards JE, Burchell HB: Effects of pulmonary hypertension of the tracheobronchial tree. Dis Chest. 1960, 38:272-284. 10.1378/chest.38.3.272

5. Perloff JK, Hart EM, Greaves SM, Miner PD, Child JS: Proximal pulmonary arterial and intrapulmonary radiologic features of Eisenmenger syndrome and primary pulmonary hypertension. Am J Cardiol. 2003, 92:182-187. 10.1016/S0002-9149(03)00535-6

6. Achouh L, Montani D, Garcia G, et al.: Pulmonary arterial hypertension masquerading as severe refractory asthma. Eur Respir J. 2008, 32:513-516. 10.1183/09031936.00005408

7. Jaijee SK, Ariff B, Howard L, O'Regan DP, Gin-Sing W, Davies R, Gibbs JS: Left main bronchus compression due to main pulmonary artery dilatation in pulmonary hypertension: two case reports. Pulm Circ. 2015, 5:723-725. 10.1086/683687

8. Lanjewar C, Shiradkar S, Agrawal A, Mishra N, Kerkar P: Aneurysmally dilated major aorto-pulmonary collateral in tetralogy of Fallot. Indian Heart J. 2012, 64:196-197. 10.1016/S0019-4832(12)60060-1

9. Morjaria S, Grinnan D, Voelkel N: Massive dilatation of the pulmonary artery in association with pulmonic stenosis and pulmonary hypertension. Pulm Circ. 2012, 2:256-257. 10.4103/2045-8932.97640

10. Żyłkowska J, Kurzyna M, Florczyk M, et al.: Pulmonary artery dilatation correlates with the risk of unexpected death in chronic arterial or thromboembolic pulmonary hypertension. Chest. 2012, 142:14061416. 10.1378/chest.11-2794

11. Kanabuchi K, Noguchi N, Kondo T: Vascular tracheobronchial compression syndrome in adults: a review . Tokai J Exp Clin Med. 2011, 36:106-111.

12. Saygili A, Aytekin C, Boyvat F, Barutçu O, Mercan S, Tokel K: Endobronchial stenting in a two-month-old infant with bronchial compression secondary to tetralogy of Fallot and absent pulmonary valve. Turk J Pediatr. 2004, 46:268-271.

13. Knauth AL, Marshall AC, Geva T, Jonas RA, Marx GR: Respiratory symptoms secondary to aortopulmonary collateral vessels in tetralogy of Fallot absent pulmonary valve syndrome. Am J Cardiol. 2004, 93:503-505. 10.1016/j.amjcard.2003.10.057

14. Takara I, Uehara M, Higa Y, Takechi A, Iha H, Sugahara K: Respiratory management in a patient with severe tracheal stenosis caused by compression from the ascending aortic arch aneurysm [Article in English, Japanese]. Masui. 2003, 52:1079-1082.

15. Ditchfield MR, Culham JA: Assessment of airways compression by MR imaging in children with aneurysmal pulmonary arteries. Pediatr Radiol. 1995, 25:190-191. 10.1007/BF02021531

16. Winn RA, Chan ED, Langmack EL, Kotaru C, Aronsen E: Dysphagia, chest pain, and refractory asthma in a 42-year-old woman. Chest. 2004, 126:1694-1697. 10.1378/chest.126.5.1694

17. Maynar M, Lopez L, Gorriz E, Reyes R, Pulido-Duque JM, Castaneda-Zuniga WR: Massive brachiocephalic artery bleeding due to a Gianturco tracheal stent. J Vasc Interv Radiol. 1993, 4:289-291.

18. Stotz WH, Berkowitz ID, Hoehner JC, Tunkel DE: Fatal complication from a balloon-expandable tracheal stent in a child: a case report. Pediatr Crit Care Med. 2003, 4:115-117.

19. Ramakrishna G, Sprung J, Ravi BS, Chandrasekaran K, McGoon MD: Impact of pulmonary hypertension on the outcomes of noncardiac surgery: predictors of perioperative morbidity and mortality. J Am Coll Cardiol. 2005, 45:1691-1699. 10.1016/j.jacc.2005.02.055 